\title{
NON-SEPARATED CUTTINGS OF CONNECTED POINT SETS*
}

\author{
BY \\ G. T. WHYBURN†
}

1. We shall consider a connected, metric and separable space which we denote by $M$. A subset $X$ of $M$ is called a cutting of $M$ provided that the complement $M-X$ of $X$ is not connected and hence is the sum of two mutually separated sets $M_{1}(X)$ and $M_{2}(X) ; X$ is said to separate two points or point sets $A$ and $B$ in $M$ when the sets $M_{1}(X)$ and $M_{2}(X)$ can be so chosen that $M_{1}(X) \supset A$ and $M_{2}(X) \supset B$, and is said to separate a single set $N$ in $M$ when $M_{1}(X)$ and $M_{2}(X)$ can be chosen so that $N \cdot M_{1}(X) \neq 0 \neq N \cdot M_{2}(X)$.

A collection $G$ of subsets of $M$ will be called non-separated provided that the elements of $G$ are mutually exclusive and no element of $G$ separates any other element of $G$ in $M$.

A subset $P$ of $M$ is said to have the potential order $\alpha$ in $M$ relative to a given collection $G$ of subsets of $M$ provided that $\alpha$ is the least cardinal number such that there exists a monotonic decreasing sequence $\left[U_{i}\right]$ of neighborhoods of $P$ such that $P=\prod_{1}^{\infty} \bar{U}_{i}$ and such that for each $i$, the boundary $F\left(U_{i}\right)$ of $U_{i}$ is a subset of the sum of $\alpha$ of the sets of the collection $G$.

In this paper we shall show, first, that if $G$ is any uncountable nonseparated collection of cuttings of $M$ then all save a countable number of the elements of $G$ have the potential order 2 in $M$ relative to $G$. Now obviously if the elements of any collection $G$ of mutually exclusive cuttings of $M$ are connected or if they reduce to single points, then the collection $G$ is nonseparated. And since for the case where $M$ is compact, the potential order of a point of $M$ is the same as its order in the Menger-Urysohn sense, our theorem yields as corollaries many important known results concerning the cut points and connected cuttings of connected sets and of continua; for example: (1) the theorem of Wazewski-Mengert that the ramification points of any acyclic continuous curve are countable, (2) the theorem of Kuratowski and Zarankiewicz $\$$ that the set of all points of any connected set $M$ whose complement in $M$ is neither connected nor the sum of two connected point

* Presented to the Society, February 22, 1930; received by the editors July 5, 1930.

$\dagger$ Fellow, John Simon Guggenheim Memorial Foundation.

† Sẹe Wazewski, Annales de la Société Polonaise de Mathématique, vol. 2 (1923), p. 49; and Menger, Fundamenta Mathematicae, vol. 10 (1927), p. 108.

$\S$ Bulletin of the American Mathematical Society, vol. 33 (1927), p. 571. 
sets is countable; (3) the theorem of the author* that all save a countable number of the cut points of any continuum are points of order 2 of $M$ in the Menger-Urysohn sense; and (4) other results concerning cuttings due to Zarankiewicz $\dagger$ and to the author.f

Second, with the aid of this theorem we shall show that if the space $M$ contains an uncountable non-separated collection $G$ of cuttings, then there exists an upper semi-continuous collection $S$ of elements such that all save a countable number of the sets of $G$ are elements of $S$ and such that every two elements of $S$ may be separated in $M$ by some third element. In case $M$ is compact, the decomposition space $S$ is an acyclic continuous curve.

Finally, we shall prove an existence theorem to the effect that every locally connected space $M$ contains an uncountable non-separated collection of cuttings. Therefore, the above mentioned decomposition is always realisable for locally connected sets $M$, and notably for the case where $M$ is a continuous curve, this decomposition gives rise to a decomposition space which is a nondegenerate acyclic continuous curve.

2. Preliminary lemmas. Let $X$ and $Y$ be any two cuttings of $M$ and set

$$
\begin{aligned}
& M-X=M_{1}(X)+M_{2}(X), \\
& M-Y=M_{1}(Y)+M_{2}(Y),
\end{aligned}
$$

representing decompositions of $M-X$ and $M-Y$ respectively into mutually separated sets. Then if $i, j, r$, and $s$ are positive integers such that $i+j=3$ $=r+s$, it follows immediately that the following equation is valid:

$$
M=M_{i}(X)+M_{r}(Y)+M_{j}(X) \cdot M_{s}(Y)+X+Y .
$$

With the aid of this equation, we deduce at once the result

(2.2) If neither of the sets $X$ and $Y$ separates the other, we may choose the indices $i$ and $r$ such that

$$
X \subset M_{r}(Y) \text { and } Y \subset M_{i}(X) ;
$$

and these relations imply also the relations

(b) $\quad M_{j}(X) \cdot M_{s}(Y)=0, M_{j}(X)+X \subset M_{r}(Y)$, and $M_{s}(Y)+Y \subset M_{i}(X)$.

Clearly this is the case, because by virtue of the relations (a) we may omit the last two terms in equation (2.1); and since $M$ is connected, the term $M_{j}(X) \cdot M_{s}(Y)$ must vanish. This fact gives at once the remaining two relations (b).

* These Transactions, vol. 30 (1928), p. 606.

† See Fundamenta Mathematicae, vol. 12 (1928), pp. 119-125.

† See Bulletin of the American Mathematical Society, vol. 35 (1929), pp. 87-104. 
Now let $G$ be any non-separated collection of cuttings of $M$ and let $E(a, b)$ be the collection of all those elements of $G$ which separate two given points $a$ and $b$ in $M$. Let $X$ and $Y$ be any two elements of $E(a, b)$ and let the indices in (i) and (ii) be chosen so that

$$
M_{1}(X) \cdot M_{1}(Y) \supset a \text { and } M_{2}(X) \cdot M_{2}(Y) \supset b .
$$

The element $X$ of $E(a, b)$ will be said to precede the element $Y$, and this fact is indicated by the notation $X<Y$, provided that for at least one set of decompositions satisfying (i), (ii) and (iii) it is true that $X \subset M_{1}(Y)$. We shall now show that this definition gives a natural order to the elements of $E(a, b)$.

First, for any two elements $X$ and $Y$ of $E(a, b)$, at least one of the relations $X<Y$ and $Y<X$ must be valid. For if $X$ does not precede $Y$, then by (2.2), (a), $r=2$ and hence $s=1$. By (b) and (iii) it follows that $j=2$ and hence $i=1$. Therefore by (a), $Y \subset M_{1}(X)$, which means $Y<X$.

Second, only one of the relations $X<Y$ and $Y<X$ can be valid. For if $X<Y$, then [for any set of decompositions whatever satisfying (i), (ii), (iii)], in (2.2), $r=1$ and hence $s=2$. By (b) and (iii) it follows that $j=1$ and hence $i=2$. Therefore by (a), $Y \subset M_{2}(X)$, which is incompatible with $Y<X$.

Finally, for any three elements $Z, X$ and $Y$ of $E(a, b)$, the relations $Z<X$, $X<Y$ imply that $Z<Y$. For then $Z \subset M_{1}(X)$ and $X \subset M_{1}(Y)$. Hence in (2.2), $r=1$ and $s=2$. By (b) and (iii) it follows that $j=1$. Therefore by the second relation in (b), $Z \subset M_{1}(X)+X \subset M_{1}(Y)$, which gives $Z<Y$.

Thus we have proved the following result:

(2.3) If each element of the non-separated collection $E(a, b)$ of subsets of $M$ separates the two points $a$ and $b$ in $M$, then the collection $E(a, b)$ possesses $a$ natural order.

For convenience we give here a lemma concerning ordered sets due to Zarankiewicz* which will be used below.

LEMMa (Zarankiewicz). If $K$ is any ordered subset of $M$, then the set $H$ of all points $p$ of $K$ such that $p$ is not at the same time a limit point of the set $P_{p}$ of all points of $K$ preceding $p$ and also of the set $F_{p}$ of all points of $K$ following $p$ is countable.

The space $M$ being separable and metric, it therefore contains a countable sequence $R_{1}, R_{2}, R_{3}, \cdots$ of open sets which is equivalent to the set of all open subsets of $M$. Now let $H_{1}$ be the set of all points of $K$ which are not limit points of their predecessors, and let $H_{2}=H-H_{1}$. For each point $p$ of $H_{1}$ let $n(p)$ be the least positive integer such that $R_{n(p)}$ contains $p$ but contains no point of $K$ which precedes $p$. Then if $p$ and $q$ are distinct points of $H_{1}$ and

* See Fundamenta Mathematicae, vol. 12 (1928), p. 119. 
$p<q$, then since $R_{n(q)}$ does not contain $p$, it follows that $n(p) \neq n(q)$, and therefore $H_{1}$ is countable. A similar argument proves $H_{2}$ countable; and hence $H$ is countable.

3. ThEOREM. If $G$ is any uncountable non-separated collection of cuttings of a connected, metric, and separable space $M$, then all save possibly a countable number of the elements of $G$ have the potential order 2 in $M$ relative to $G$.

Suppose, on the contrary, that $G$ contains an uncountable subcollection $G_{1}$ no element of which has the potential order 2 in $M$ relative to $G$. Now there exist two points $a$ and $b$ of $M$ such that the collection $E(a, b)$ of all those elements of $G_{1}$ which separate $a$ and $b$ in $M$ is uncountable; for $M$ being separable, there exists a countable subset $D$ of $M$ such that $\bar{D}=M$; and since every element of $G_{1}$ which contains no point of $D$ must separate some pair of points of $D$ in $M$, and since the set of all pairs of points of $D$ is countable, it follows that for at least one pair of points $a, b$ of $D$, the set $E(a, b)$ is uncountable.

By $\$ 2$ the elements of the collection $E(a, b)$ possess a natural order; and if $K$ is a point set which contains exactly one point $x$ of each element $X$ of $E(a, b)$ and contains no other points, then $K$ is an ordered point set. Indeed for each pair $x, y$ of points of $K$, set $x<y$ provided that $X<Y$. By the Zarankiewicz lemma, the set $H$ of all points $p$ of $K$ which are not at the same time a limit point both of their predecessors and of their successors is countable. Let $H(a, b)$ be the collection of all those sets $X$ of $E(a, b)$ such that the corresponding point $x$ in $K$ belongs to $K-H$. Then $H(a, b)$ is uncountable and each element $X$ of $H(a, b)$ contains a point $x$ which is a limit point of the sum of the predecessors of $X$ and also of the sum of the successors of $X$.

Now for each element $X$ of $H(a, b)$, there exist mutually separated sets $M_{1}(X)$ and $M_{2}(X)$ such that

$$
M-X=M_{1}(X)+M_{2}(X), M_{1}(X) \supset a \text { and } M_{2}(X) \supset b .
$$

And with the aid of what has just been shown it follows immediately that there exist two infinite sequences of elements $X_{1}, X_{2}, X_{3}, \cdots$ and $Y_{1}, Y_{2}$, $Y_{3}, \cdots$ of $H(a, b)$ such that, for each $n$,

$$
X_{n}<X_{n+1}<X<Y_{n+1}<Y_{n},
$$

and such that $X$ contains a point which is a limit point both of $\sum X_{n}$ and $\sum Y_{n}$.

Since by supposition no element of $H(a, b)$ can have the potential order 2 in $M$ relative to $G$, it follows that if for each element $X$ of $H(a, b), V_{n}(X)$ denotes the set of points $M-\left[M_{1}\left(X_{n}\right)+M_{2}\left(Y_{n}\right)\right]$, then there exists at least one point $p_{x}$ belonging to the point set 


$$
\prod_{1}^{\infty} V_{n}(X)-X
$$

for if this were not the case, then by virtue of (1) and equation (2.1) in which substitute $X_{n}$ for $X, Y_{n}$ for $Y, 1$ for $i$ and 2 for $r$, it follows that $V_{n}(X)$ $\supset M_{2}\left(X_{n}\right) \cdot M_{1}\left(Y_{n}\right) \supset X$; and if for each point $p$ of $M_{2}\left(X_{n}\right) \cdot M_{1}\left(Y_{n}\right)$ we take a neighborhood $N_{p}$ of $p$ of diameter less than $1 / 4$ the distance from $p$ to the set of points $\overline{M_{1}\left(X_{n}\right)}+\overline{M_{2} Y(n)}$, and call $U_{n}(X)$ the sum of all the neighborhoods $N_{p}$, then it follows readily that

$$
X \subset M_{2}\left(X_{n}\right) \cdot M_{1}\left(Y_{n}\right) \subset U_{n}(X) \subset \overline{U_{n}(X)} \subset V_{n}(X) ;
$$

and hence $F\left[U_{n}(X)\right] \subset X_{n}+Y_{n}, U_{n}(X) \subset U_{n-1}(X)$ and $X=\prod_{1}^{\infty} \overline{U_{n}(X)}$; but then $X$ has the potential order 2 in $M$ relative to $G$, contrary to supposition.

Now if $X$ and $Y$ are any two elements of $H(a, b), X \neq Y$, it follows that $p_{x} \neq p_{y}$. For suppose $X<Y$. Then since $X$ contains a limit point of the sum of its successors in $E(a, b)$ but contains no limit point of $M_{2}(Y)$, it follows that there exist two elements $Y_{k}$ and $Y_{m}$ in the " $Y$-sequence" in (1) for the element $X$ such that

$$
X<Y_{k}<Y_{m}<Y ;
$$

and since $Y$ contains a limit point of the sum of its predecessors in $E(a, b)$ but contains no limit point of $M_{1}\left(Y_{m}\right)$, it follows that there exists an element $X_{n}$ of the " $X$-sequence" for $Y$ in (1) such that

$$
X<Y_{k}<Y_{m}<X_{n}<Y .
$$

Consequently it follows with the aid of (2.2) that

$$
p_{x} \subset M_{1}\left(Y_{k}\right)+Y_{k} \subset M_{1}\left(Y_{m}\right)
$$

and

$$
p_{y} \subset M_{2}\left(X_{n}\right)+X_{n} \subset M_{2}\left(Y_{m}\right),
$$

and hence $p_{x} \neq p_{y}$.

Now let $L$ denote the set of all points $\left[p_{x}\right]$ for all elements $X$ of $H(a, b)$. Then $L$ is uncountable and is an ordered set; indeed, it is only necessary to set $p_{x}<p_{y}$ when $X<Y$. Therefore by the Zarankiewicz lemma, there exists a point $p_{x}$ of $L$ which is a limit point both of its predecessors and of its followers, and hence both of $\sum X_{n}$ and of $\sum Y_{n}$, where the sequences $\left[X_{n}\right]$ and $\left[Y_{n}\right]$ satisfy (1). But $\sum X_{n} \subset M_{1}(X)$ and $\sum Y_{n} \subset M_{2}(X)$; and $p_{x}$ must then belong either to $M_{1}(X)$ or to $M_{2}(X)$ and be a limit point of the other, contrary to the fact that these two sets are mutually separated. Thus the supposition that our theorem is false leads to a contradiction. 
4. Consequences of $\S 3$. Let $G$ be any uncountable non-separated collection of cuttings of $M$. Then since the product of any family [ $\left.\bar{U}_{n}\right]$ of closed sets is closed, $\$ 3$ yields at once the result

( $\alpha)$ All save a countable number of the elements of $G$ are closed point sets.

Now if $X$ is any element of $G$ such that $M-X$ is not the sum of two connected point sets, $X$ cannot have a potential order 2 in $M$ relative to $G$. For $M-X=M_{1}(X)+M_{2}(X)+M_{3}(X)$, where the sets $M_{1}(X), M_{2}(X)$, and $M_{3}(X)$ are mutually separated and contain points $a_{1}, a_{2}$ and $a_{3}$ respectively; and if $X$ had the potential order 2 relative to $G$, there would exist two elements $X_{1}$ and $X_{2}$ of $G$ and a neighborhood $U$ of $X$ such that $F(U) \subset X_{1}+X_{2}, X_{1} \subset M_{1}(X)$, $X_{2} \subset M_{2}(X)$ and $\bar{U} \cdot\left(a_{1}+a_{2}+a_{3}\right)=0$; but then it would readily follow that the point set $M_{3}(X) \cdot(M-\bar{U})$ is non-vacuous and is both open and closed, contrary to the fact that $M$ is connected. Thus in consequence of the theorem in $\$ 3$ we have

( $\beta$ ) The complement of each element of $G$, with the exception of a countable number of such elements, consists of exactly two components.

Let us denote by $\rho$ the property of any subset $N$ of $M$ not to be separated in $M$ by any single element of $G$. Clearly each element $X$ of $G$ has the property $\rho$. We shall now show that

$(\gamma)$ All save a countable number of the elements of $G$ are saturated in $M$ relative to the property $\rho$.

If, on the contrary, $G$ contains an uncountable subcollection $G_{1}$ no element of which is saturated relative to the property $\rho$, then for each element $Z$ of $G_{1}$ there exists at least one point $p_{z}$ which is not separated from $Z$ in $M$ by any single element of $G$. Under these conditions it follows by the theorem and proof in $\$ 3$ that there exist two points $a$ and $b$ of $M$ and three elements $Z, X$ and $Y$ of $E(a, b)$ (the collection of all those elements of $G_{1}$ which separate $a$ and $b$ ) such that $X<Z<Y$, and $M_{2}(X) \cdot M_{1}(Y)$ contains $Z$ but does not contain the point $p_{z}$ and also such that $X+Y$ does not contain $p_{z}$. But then by equation (2.1) we have either $p_{z} \subset M_{1}(X)$ or $p_{z} \subset M_{2}(Y)$. This is impossible because in the first case $X$ separates $p_{z}$ and $Z$ in $M$ and in the second case $Y$ separates $p_{z}$ and $Z$ in $M$.

A cutting $X$ of $M$ is said to be an irreducible cutting of $M$ provided that no proper subset of $X$ is a cutting of $M$.

( $\delta)$ All save a countable number of the elements of $G$ are irreducible cuttings of $M$.

If this is not so, there exists an uncountable collection $G^{0}$ of cuttings of $M$ such that for each element $X^{0}$ of $G^{0}$ there exists an element $X$ of $G$ and a point $p_{x}$ of $X$ such that $X^{0} \subset X-p_{x}$. Since $G$ is non-separated, it follows at once that $G^{0}$ is non-separated. Therefore by $(\gamma)$ there exists an element $X^{0}$ 
of $G^{0}$ which is saturated relative to the property $\rho$ defined by the collection $G^{0}$. Consequently there exists an element $Y^{0}$ of $G^{0}$ which separates $X^{0}$ and $p_{x}$ in $M$, and one has $M-Y^{0}=M_{1}\left(Y^{0}\right)+M_{2}\left(Y^{0}\right)$, where $M_{1}\left(Y^{0}\right) \supset X^{0}$ and $M_{2}\left(Y^{0}\right) \supset p_{x}$. But then $M-Y=M_{1}\left(Y^{0}\right) \cdot(M-Y)+M_{2}\left(Y^{0}\right) \cdot(M-Y)$, and thus $Y$ separates $X$ in $M$ (for $\left.Y \cdot\left(X^{0}+p_{x}\right) \subset Y \cdot X=0\right)$, which contradicts the non-separatedness of $G$.

We prove now the following general theorem:

THEOREM. Every uncountable non-separated collection $G$ of cuttings of a connected, metric, and separable space $M$ contains a subcollection $Q$ which contains all save possibly a countable number of the elements of $G$ and such that each element $X$ of $Q$ has the following properties: (a) $X$ is closed; (b) $M-X$ is the sum of two mutually separated connected point sets; (c) $X$ is saturated in $M$ relative to the property $\rho$ defined by the collection $Q$, i.e., for every point $p$ of $M-X$, there exists an element $Y$ of $Q$ which separates $X$ and $p$ in $M$; (d) $X$ is an irreducible cutting of $M$; and (e) $X$ has the potential order 2 in $M$ relative to $Q$.

To obtain the collection $Q$, let $D$ be a countable subset of $M$ which is dense in $M$ and let us omit from $G$ : (1) every element which does not possess each of the properties (a), (b), and (d); (2) every element which separates in $M$ some pair of points $a, b$ of $D$ which are separated by only a countable number of elements of $G$; (3) every element which separates some pair $a, b$ of points of $D$ and contains no point $p$ having the property that every neighborhood of $p$ contains points of uncountably many distinct elements of $G$ which separate $a$ and $b$. Let $G_{1}$ denote the collection of the elements of $G$ remaining after these omissions. Then by virtue of $(\alpha),(\beta)$ and $(\delta)$, together with the facts that there are only a countable number of pairs of points of $D$ and that in the space $M$ every uncountable set of points contains a point of condensation of itself, it follows that $G_{1}$ contains all save possibly a countable number of the elements of $G$.

Now let us omit from $G_{1}$ every element which is not saturated in $M$ relative to the property $\rho$ defined by the collection $G_{1}$ and also every element which does not have the potential order 2 in $M$ relative to $G_{1}$. Let $Q$ be the collection of elements of $G_{1}$ remaining after these omissions. Then $Q$ contains all save a countable number of the elements of $G_{1}$ and hence also of $G$, and every element $X$ of $Q$ has the desired properties (a)-(e). Clearly $X$ has properties (a), (b) and (d), for every element of $G_{1}$ has these properties. It remains to show that $X$ has properties (c) and (e).

To show that $X$ has property (c), let $p$ be any point of $M-X$. There exists an element $Y$ of $G_{1}$ which separates $X$ and $p$, because every element of $Q$ is saturated in $M$ relative to the property $\rho$ defined by $G_{1}$. Hence $M-Y$ 
$=M_{1}(Y)+M_{2}(Y)$, where $M_{1}(Y) \supset X$ and $M_{2}(Y) \supset p$. Also $M-X=M_{1}(X)$ $+M_{2}(X)$, where $M_{2}(X) \supset Y$. Thus if $a$ and $b$ are points of $M_{1}(X)$ and $M_{2}(Y)$ respectively belonging to $D$, both $X$ and $Y$ separate $a$ and $b$ in $M$, and we have $X<Y$ in the order from $a$ to $b$. Now there exists also an element $Z$ of $G_{1}$ which separates $X$ and $Y$ in $M$, and it follows from $\$ 2$ that $Z$ also separates $a$ and $b$ in $M$, and we have the order $X<Z<Y$. Thus $Z \subset M_{2}(X) \cdot M_{1}(Y)$. Since $X$ and $Y$ are closed, $M_{2}(X) \cdot M_{1}(Y)$ is a neighborhood of $Z$, and hence it contains points of (and therefore contains all of) uncountably many elements of $G$ which separate $a$ and $b$ in $M$. Therefore there exists at least one of these elements, say $W$, which belongs to $Q$, for all but a countable number of the elements of $G$ belong to $Q$. Thus we have the order $X<W<Y$; and since $p \subset M_{2}(Y)$, it follows that $W$ separates $X$ and $p$ in $M$. Consequently $X$ has property (c).

Since $X$ has the potential order 2 in $M$ relative to $G_{1}$, there exist, as shown in $\S 3$, two points $a$ and $b$ of $M$ such that $X$ belongs to the collection $E_{(}^{\prime}(a, b)$ of all those elements of $G_{1}$ which separate $a$ and $b$ in $M$ and such that there exist two sequences $X_{1}, X_{2}, \cdots$ and $Y_{1}, Y_{2}, \cdots$ of elements of $E(a, b)$ so that

$$
X_{n}<X_{n+1}<X<Y_{n+1}<Y_{n}
$$

and such that if $U_{n}=M_{2}\left(X_{n}\right) \cdot M_{1}\left(Y_{n}\right)$, then $X=\prod_{1}^{\infty} \bar{U}_{n}$. Now for each $n$ there exist, by virtue of property (c), two elements $X_{n}^{\prime}$ and $Y_{n}^{\prime}$ of $Q$ belonging to $E(a, b)$ such that $X_{n}<X_{n}^{\prime}<X<Y_{n}^{\prime}<Y_{n}$. Hence if $U_{n}^{\prime}$ denotes the point set $M_{2}\left(X_{n}^{\prime}\right) \cdot M_{1}\left(Y_{n}^{\prime}\right)$, one has $U_{n}^{\prime} \subset U_{n}$. Hence $X=\Pi_{1}^{\infty} \bar{U}_{n}^{\prime}$, and since $F\left(U_{n}^{\prime}\right)$ $c X_{n}^{\prime}+Y_{n}^{\prime}$ and since clearly the sequence $\left[U_{n}\right]$ contains an infinite subsequence $\left[U_{n_{i}}\right.$ ] such that $U_{n_{i+1}} \subset U_{n_{i}}$, it follows that $X$ has the potential order 2 in $M$ relative to $Q$. This completes the proof.

5. Decomposition of $M$ by means of a non-separated collection $G$ every element of which is saturated relative to property $\rho$. Let $G$ be any non-separated collection of subsets of $M$ each of which is saturated in $M$ relative to the property $\rho$ defined by $G$. For each point $e$ of $M$ which belongs to no element of $G$, let $E$ denote the point set consisting of $e$ together with all points $p$ of $M$ which are not separated in $M$ from $e$ by any single element of $G$. Let $S$ denote the collection whose elements are the elements of $G$ together with all such point sets $E$ thus defined. Clearly each element of $S$ is closed and every point of $M$ belongs to exactly one element of $S$. We shall show next that the collection $S$ is non-separated.

Suppose, on the contrary, that some element $X$ of $S$ separates some pair of points $p$ and $q$ belonging to an element $Y$ of $S$. Then $M-X=M_{1}(X)$ $+M_{2}(X)$, where $M_{1}(X) \supset p$ and $M_{2}(X) \supset q$. Now by virtue of the definition of the collections $G$ and $S$, it follows that there exists an element $Z$ of $G$ which 
separates $X$ and $p$ in $M$. Hence $M-Z=M_{1}(Z)+M_{2}(Z)$, where $M_{1}(Z) \supset X$ and $M_{2}(Z) \supset p$. Since $Z$ belongs to $G$, it cannot separate $Y$ in $M$; and therefore $p+q \subset Y \subset M_{2}(Z)$. But then

$$
M-Z=\left[M_{1}(Z)+M_{1}(X) \cdot M_{2}(Z)\right]+M_{2}(X) \cdot M_{2}(Z),
$$

and we have a separation of $M-Z$ into two mutually separated sets containing the points $p$ and $q$ respectively of $Y$, contrary to the fact that since $Z$ belongs to $G$ it cannot separate $Y$ in $M$. Therefore $S$ is non-separated.

Now clearly every element of $S$ is saturated in $M$ relative to the property $\rho$ defined by the collection $S$. Consequently every two elements $X$ and $Y$ of $S$ are separated in $M$ by some third element of $S$. With the aid of this property it follows immediately that the collection $S$ is upper semi-continuous, ${ }^{*}$ i.e., there does not exist a sequence $X_{1}, X_{2}, X_{3}, \cdots$ of elements of $S$ and two sequences $\left[p_{i}\right]$ and $\left[q_{i}\right]$ of points such that $p_{i}+q_{i} \subset X_{i}$ and which have sequential limit points $p$ and $q$ respectively belonging to two different elements $P$ and $Q$ respectively of $S$. For there exists an element $X$ of $S$ such that $M-X$ $=M_{1}(X)+M_{2}(X)$ where $M_{1}(X) \supset P$ and $M_{2}(X) \supset Q$; and since for each $i, X_{i}$ is a subset either of $M_{1}(X)$ or of $M_{2}(X)$, either $M_{1}(X)$ or $M_{2}(X)$ contains $X_{i}$ for infinitely many $i$ 's; but this is impossible, for both $p$ and $q$ are limit points of every infinite subsequence of $X_{1}, X_{2}, X_{3}, \cdots$.

Now in case the space $M$ is compact, the elements of $S$ are closed and compact, and if for each pair of elements $\dot{X}$ and $Y$ of $S$ we define the distance $\rho(X, Y)$ between $X$ and $Y$ as the upper limit of the distances $\rho(x, y)$, where $x$ and $y$ are points of $X$ and $Y$ respectively, it readily follows that the space $S^{\prime}$ so obtained is compact, separable, metric and connected; and since it readily follows that every two "points" of $S^{\prime}$ are separated in $S^{\prime}$ by some third "point" of $S^{\prime}$, therefore $S^{\prime}$ is an acyclic continuous curve.

6. Existence Theorem. If the space $M$ is connected im kleinen, there exists an uncountable non-separated collection of cuttings of $M$.

Let $a$ and $b$ be any two points of $M$, and for each positive number $r$ which is less than the distance from $a$ to $b$, let $S(a, r)$ denote the set of all points of $M$ whose distance from $a$ is equal to $r$ and let $I(a, r)$ denote the set of all points at a distance $<r$ from $a$. Then for each $r, S(a, r)$ separates $a$ and $b$ in $M$. Let $R(a, r)$ denote the component of $M-S(a, r)$ containing $a$, let $R(b, r)$ denote the component of $M-\overline{R(a, r)}$ containing $b$, and let $X_{r}$ denote the point set $\overline{R(a, r)} \cdot \overline{R(b, r)}$. Then clearly $X_{r}$ separates $a$ and $b$ in $M$ and we have

$$
\begin{gathered}
X_{r} \subset F[R(a, r)] \subset S(a, r), \text { and } X_{r}=F[R(b, r)] ; \\
R(a, r) \subset I(a, r) .
\end{gathered}
$$

* See R. L. Moore, these Transactions, vol. 27 (1925), pp. 416-428.

† See R. L. Moore, Fundamenta Mathematicae, vol. 7 (1925), pp. 302-307. 
Obviously the collection of cuttings $\left[X_{r}\right]$ is uncountable. It remains to show that it is non-separated. Let $X_{r_{1}}$ and $X_{r_{2}}$ be any two elements of this collection and suppose $r_{1}<r_{2}$. By (ii) it follows that $\overline{R\left(a, r_{1}\right)} \subset R\left(a, r_{2}\right)$. Thus $X_{r_{1}} \subset R\left(a, r_{2}\right)$, and therefore $X_{r_{2}}$ does not separate $X_{r}$ in $M$. From the inclusion $\overline{R\left(a, r_{1}\right)} \subset R\left(a, r_{2}\right)$ and (i) it follows that $\overline{R\left(b, r_{2}\right)}=R\left(b, r_{2}\right)+X_{r_{2}} \subset R\left(b, r_{1}\right)$, and consequently $X_{r}$ does not separate $X_{r_{2}}$ in $M$. Thus the collection $\left[X_{r}\right]$ is non-separated, and the theorem is proved.

Now since $a$ may be any point whatever of $M$ and since every neighborhood of $a$ contains uncountably many of the sets $\left[X_{r}\right]$, it follows by $\$ 4,(\delta)$, that every such neighborhood contains at least one set $X_{r}$ which is an irreducible cutting of $M$. Thus we have the following

COROLlaRY. Every open subset of a connected and connected im kleinen point set $M$ lying in a separable metric space contains an irreducible cutting $I$ of $M$.

This corollary answers a question raised by the author.*

As a result of this existence theorem it follows that the decomposition treated in $\$ 5$ is always realisable in case $M$ is locally connected; and in case $M$ is a continuous curve, $M$ may be decomposed upper semi-continuously into a collection $S$ of the type attained in $\S 5$, and the decomposition space $S^{\prime}$ is a non-degenerate acyclic continuous curve.

7. Concluding remarks. Although it is easily seen with the aid of a very simple example that two cuttings $X$ and $Y$ of $M$ may have the property that neither of them separates the other in $M$ and yet the set $M_{2}(X)+X$ not be connected, where $M_{2}(X) \supset Y$, nevertheless the following lemma is true.

LEMma. If $a$ and $b$ are two points of $M$ and $X_{1}, X_{2}, X_{3}, \cdots$ is any infinite sequence of distinct mutually exclusive sets each of which separates $a$ and $b$ in $M$ and no one of which separates any other one, and we have

$$
X_{1}<X_{2}<X_{3}<\cdots \text {, }
$$

then the set of points $\sum_{1}^{\infty} M_{1}\left(X_{i}\right)$ is connected.

For if on the contrary this set of points is the sum of two mutually separated sets $N_{1}$ and $N_{2}$, then since $a$ belongs to all of the sets $M_{1}\left(X_{i}\right)$, there exists an integer $n$ such that $N_{1} \cdot M_{1}\left(X_{n}\right) \neq 0 \neq N_{2} \cdot M_{1}\left(X_{n}\right)$. Since by (2.2), (b), it follows that $M_{1}\left(X_{n}\right) \subset M_{1}\left(X_{n+1}\right)$, therefore $N_{1} \cdot M_{1}\left(X_{n+1}\right) \neq 0 \neq N_{2} \cdot M_{1}\left(X_{n+1}\right)$. Since these two sets are mutually separated, one of them, say $N_{1} \cdot M_{1}\left(X_{n+1}\right)$,

* See Fundamenta Mathematicae, vol. 13 (1929), p. 50, where the question is raised for continuous curves $M$. A solution of this problem for the case where $M$ is a plane continuous curve has been given by J. H. Roberts; see these Transactions, vol. 32 (1930), p. 19. 
contains $X_{n}$. But then it is easily seen that the sets $N_{2} \cdot M_{1}\left(X_{n}\right)$ and $M-N_{2}$ - $M_{1}\left(X_{n}\right)$ are mutually separated, contrary to the fact that $M$ is connected.

With the aid of this lemma it can be shown without great difficulty that if $X$ is any element of a non-separated collection $G$ of subsets of $M$ which is saturated in $M$ relative to the property $\rho$ defined by the collection $G$, then

(1) each component of $M-X$ is open in $M$;

(2) the components of $M-X$ are countable;

(3) $X$ is a potentially regular element of $G$ in $M$ relative to $G$, i.e., a monotone decreasing sequence of neighborhoods $\left[U_{i}\right]$ of $X$ exists such that $F\left(U_{i}\right)$ is a subset of a finite number of the elements of $G$ and $X=\Pi_{1}^{\infty} \bar{U}_{i}$;

(4) the potential order of $X$ in $M$ relative to $G$ is equal to the number of components of $M-X$ when this number is finite, and is equal to $\omega$ (i.e., $X$ is of increasing order) when and only when this number is infinite.

JoHns Hopkins UNIVERSITY, BALTTMORE, MD. 